\title{
Germanica
}

$47 \mid 2010$

« Krack ! Tschock ! Pflatsch ! Bummmm ! La BD de langue allemande (à suivre...) »

\section{Wilhelm Busch, si loin, si proche : quelques remarques sur l'un des fondateurs de la BD moderne}

In der Ferne so nah. Einige Bemerkungen über einen der Begründer der modernen Comics.

Wilhelm Busch so far and yet so near. Some remarks on one of the founding fathers of the modern Graphic Novel.

\section{Richard Parisot}

\section{OpenEdition}

\section{Journals}

Édition électronique

URL : http://journals.openedition.org/germanica/1106

DOI : 10.4000/germanica. 1106

ISSN : 2107-0784

\section{Éditeur}

Université de Lille

\section{Édition imprimée}

Date de publication : 31 décembre 2010

Pagination : 25-36

ISBN : 9782913857261

ISSN : 0984-2632

\section{Référence électronique}

Richard Parisot, «Wilhelm Busch, si loin, si proche : quelques remarques sur l'un des fondateurs de la BD moderne », Germanica [En ligne], 47 | 2010, mis en ligne le 01 décembre 2012, consulté le 06 octobre 2020. URL : http://journals.openedition.org/germanica/1106 ; DOI : https://doi.org/10.4000/ germanica. 1106

Ce document a été généré automatiquement le 6 octobre 2020.

() Tous droits réservés 


\title{
Wilhelm Busch, si loin, si proche : quelques remarques sur l'un des fondateurs de la BD moderne
}

\author{
In der Ferne so nah. Einige Bemerkungen über einen der Begründer der \\ modernen Comics. \\ Wilhelm Busch so far and yet so near. Some remarks on one of the founding \\ fathers of the modern Graphic Novel.
}

\section{Richard Parisot}

1 Présentée en 2008 au musée Wilhelm Busch de Hanovre, l'exposition célébrant le centenaire de la mort de l'artiste mettait en lumière les multiples facettes de sa personnalité ${ }^{1}$. Les questions que posait cette exposition concernaient le «véritable " Wilhelm Busch et l'origine de son imagination débordante. Autant de petites énigmes qui préoccupent les lecteurs de Busch depuis un siècle et demi environ. "Wilhelm Busch critiquait à la fois ses semblables et la société de son époque ", rappelait à cette occasion Hans Joachim Neyer, directeur du musée. En fait ces " histoires illustrées » s'inspiraient principalement, au départ en tout cas, de la vie quotidienne rurale dans sa région natale de Basse-Saxe. Originaire de Wiedensahl, près de Hanovre, où il naquit le 15 avril 1832 (année, faut-il y voir un signe? de la mort de Goethe !), ce fils d'épicier fit des études d'art à Dusseldorf, Anvers et Munich. En 1858 il se distingue par son travail pour les Fliegende Blätter, édités par Braun et Schneider à Munich². Mais c'est en 1865 que parut Max und Moritz,suite de dessins accompagnés de ses propres vers, relatant les mauvais tours de deux garnements ${ }^{3}$. 


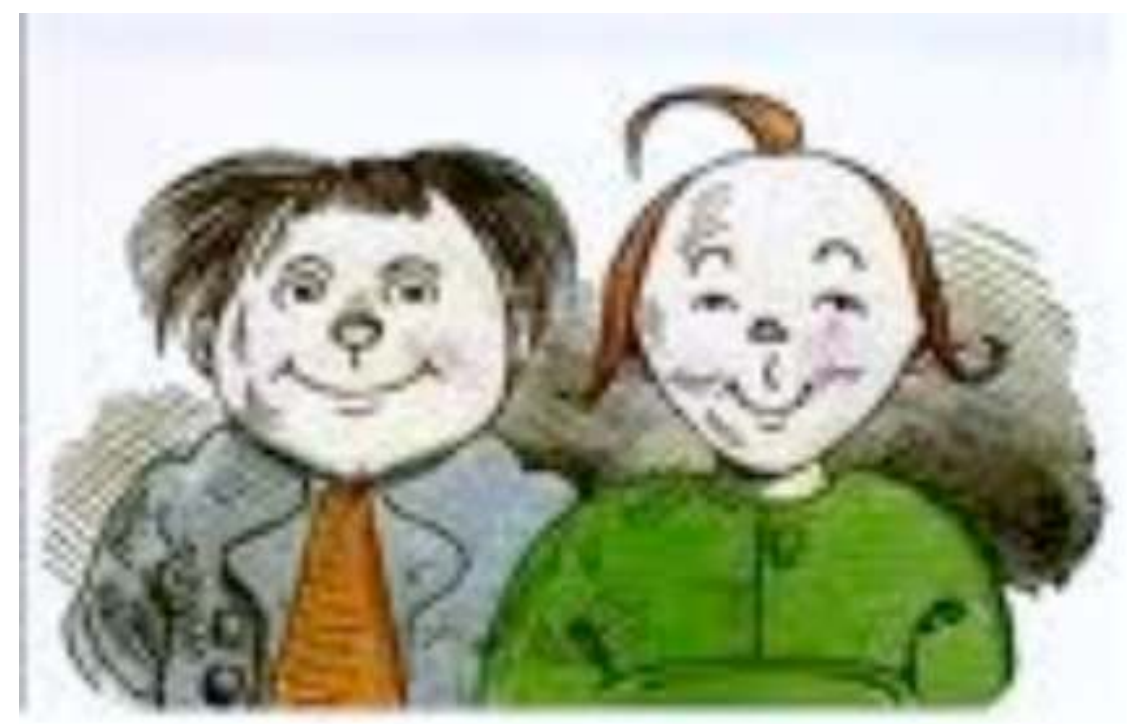

2 Cet ouvrage eut un succès mondial, faisant de son auteur un des ancêtres incontestables de la bande dessinée. Wilhelm Busch continua ensuite dans la même veine, ce qui donna, outre l'histoire de la pieuse Hélène, celle du peintre Klecksel (qui finit par se faire aubergiste) et les malheurs de Balduin Bählamm, le poète qui ne trouve pas le repos désiré à la campagne. Dans toutes ces œuvres, Busch « prend pour cible son environnement campagnard qu'il soumet à son regard acéré et ironique ainsi qu'à son humour parfois caustique: enfants mal élevés, couples bagarreurs, bigotes hypocrites, sans oublier les tortionnaires d'animaux $»^{4}$. Son point fort n'est pas la compassion mais bien davantage l'esprit satirique. On a rapidement remarqué que, par la liberté, la nervosité, la spontanéité et la sûreté de son trait, Busch se situait dans la ligne du Suisse francophone Rodolphe Töpffer, pionnier de l'esquisse non retouchée et de la bande dessinée moderne. Tout cela explique peut-être l'influence que l'humour de Wilhelm Busch a exercée plus tard sur des auteurs de bande dessinée new-yorkais et même sur des réalisateurs à Hollywood. Au début du xx siècle, ils copièrent en effet des éléments caractéristiques de son œuvre, par exemple les bras et les jambes emmêlés. De là à voir en Wilhelm Busch le "précurseur de la bande dessinée moderne », il n'y a qu'un pas.

Mais un autre aspect jusqu'à présent méconnu du travail de Wilhelm Busch, la peinture de paysages, restait, et reste peut-être encore, à découvrir, même si les illustrations proposées par Gudrun Schury contribuent à nous instruire sur ce point ${ }^{5}$. Ce qui est étonnant, mais seulement dans une certaine mesure, c'est que le travail de Busch ne soit pas étudié de la même manière de chaque côté du Rhin. En 2007-2008 sont parus, en Allemagne, quantité de textes commentant la vie et les œuvres du dessinateur ${ }^{6}$, peu de chose en France depuis la publication, en 1980, par les éditions Pierre Horay, d'un très bel album consacré à Busch, avec une préface traduite de l'allemand par Sylvia Gourdet et Caroline Kruse.

Quand on s'interroge sur le talent, le génie et le succès de l'auteur, on ne peut pas ne pas penser immédiatement à un certain nombre de facteurs et de traits caractéristiques comme une nette simplification (qui sait préserver l'amour du détail en continuant à apporter beaucoup de soin au dessin des attitudes), un goût prononcé pour le geste et le mouvement (ce qui évite l'aspect figé de certains cartons d'autres auteurs du XIX ${ }^{\mathrm{e}}$ siècle) et bien sûr une critique acerbe qui s'exprime de manière discrète parfois, à 
peine suggérée, et, à d'autres endroits, de façon très explicite. Nous ne pourrons mettre ici en lumière que quelques aspects de cet immense talent en insistant peut-être davantage sur la critique sociale au sens large. Il convient également de mentionner que Busch développe l'idée de la série. Ses dessins assortis de textes constituent bien des histoires originales, mais certaines de ces histoires auront des suites, que les lecteurs attendront, comme la BD et le manga d'aujourd'hui.

L'efficacité de ses petites histoires (Max und Moritz, 1865 ; Tobias Knopp, 1876 ; Plisch und Plum, 1882) est due à un remarquable pouvoir de simplification. Busch aime planter son décor, un peu comme au théâtre. Il nous montre les éléments qui, peu ou prou, tôt ou tard, joueront un rôle dans l'histoire. Un héritage du réalisme français et/ou allemand ? On pourra en voir ci-dessous un exemple tiré du dritter Streich ${ }^{7}$ de Max und Moritz :

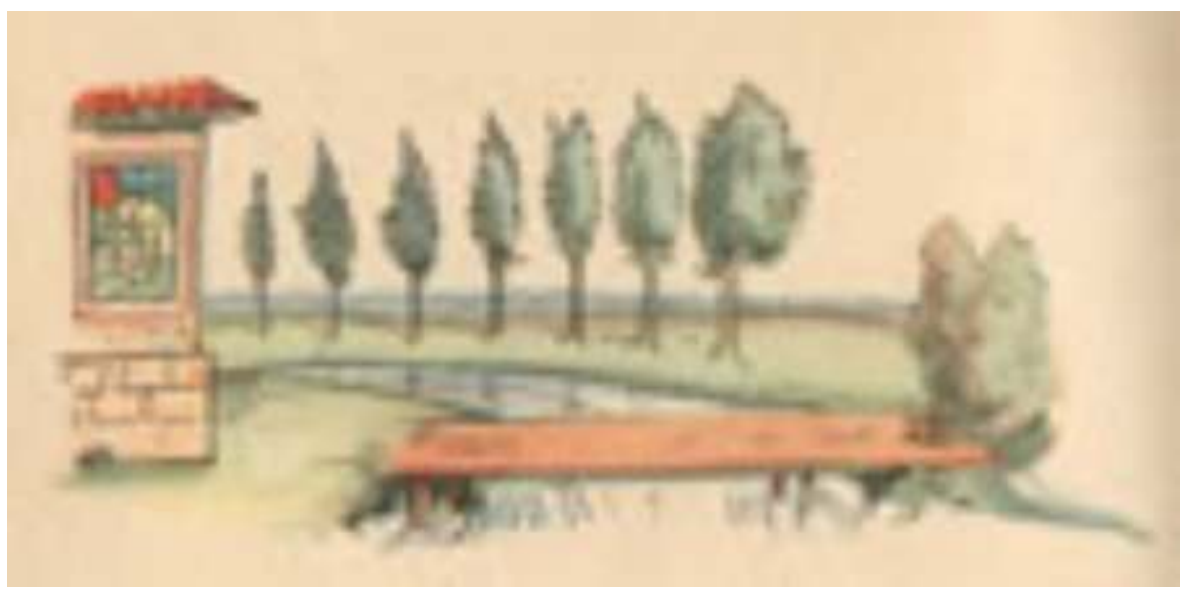

Ce décor va s'animer de façon inopinée parfois par suite de l'irruption de personnages (parfois de créatures du règne animal), toujours grotesques et caricaturaux. L'ordre, la tranquillité du décor original (originel?) est comme la condition préalable au déroulement (déchaînement?) de l'action.

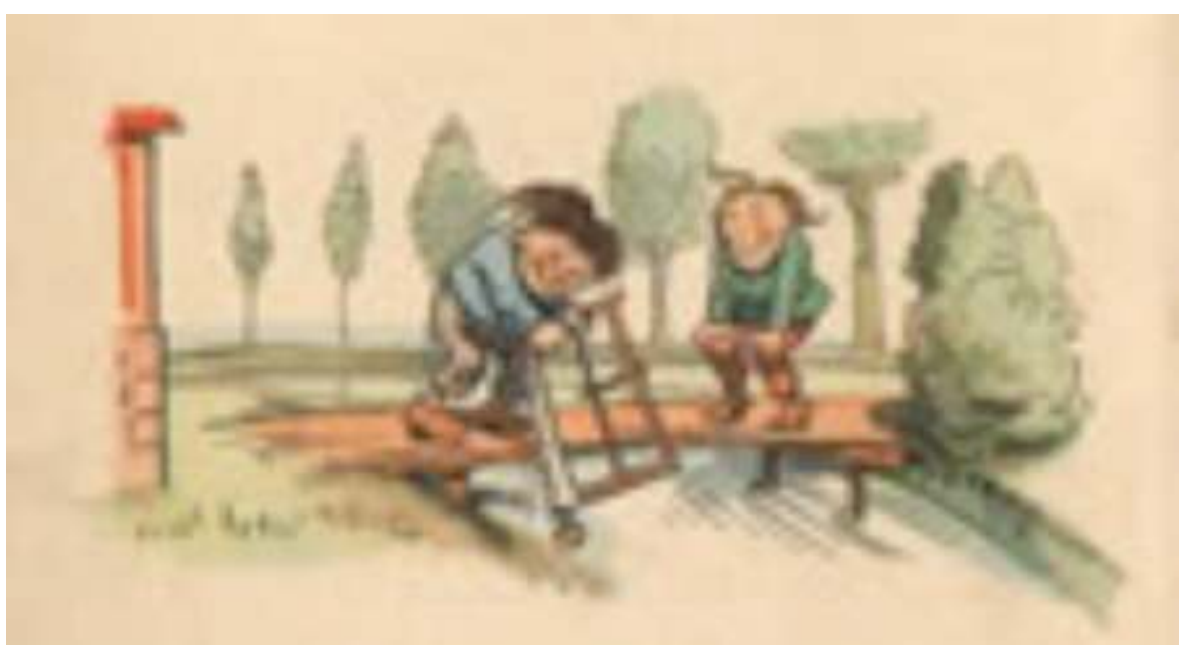

7 Cette action, vite portée à son comble, prend toujours des allures destructrices. Les deux héros favoris de Busch, Max et Moritz, se livrent à des «tours " qui débouchent sur le mauvais goût et la cruauté. L'artiste tire avant tout parti du mouvement, aux dépens de l'invention narrative. Rien ne vient atténuer la sauvagerie de certains faits !!! 

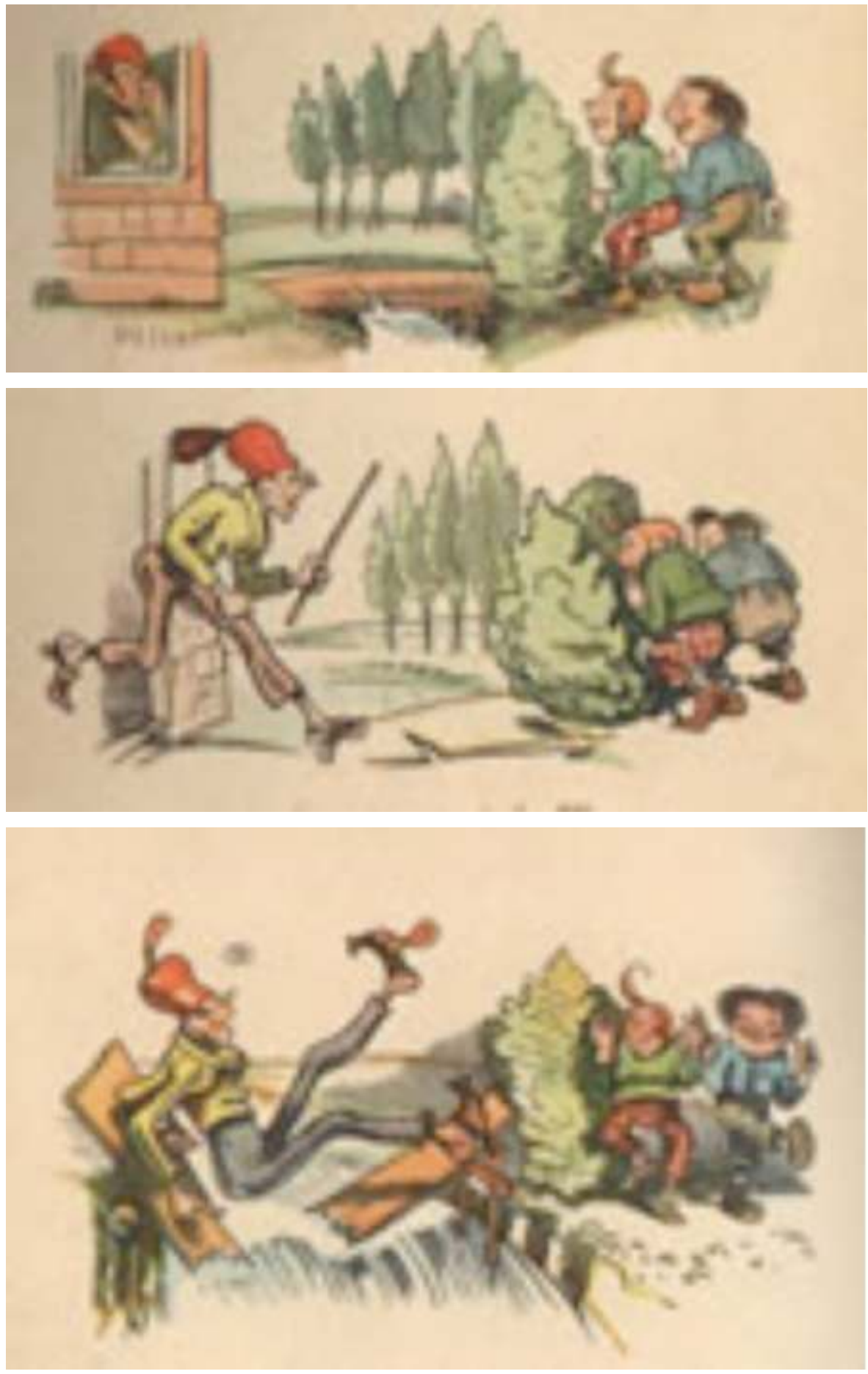


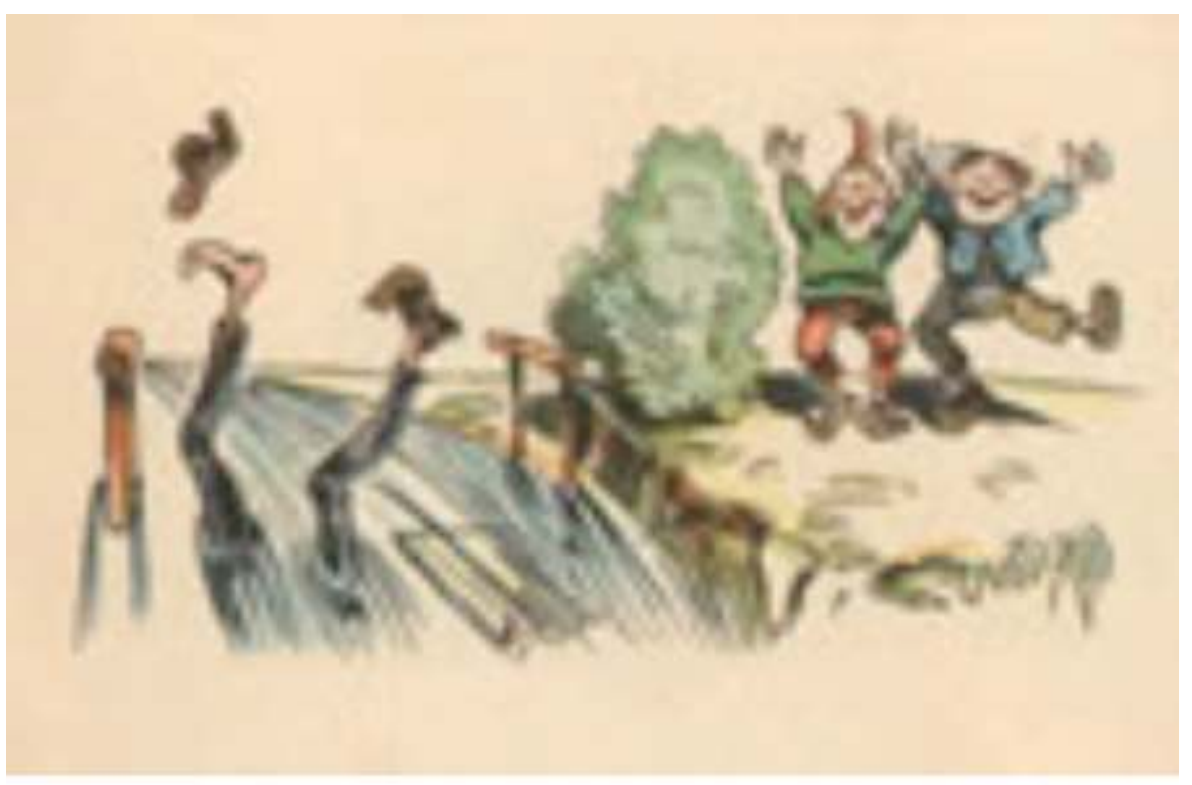
et les personnages bien-pensants prennent toujours le ciel à témoin. Libre au lecteur d'imaginer le pire. C'est vrai qu'elle est fortement catéchisée, cette jeune âme, par exemple par son oncle et sa tante : on peut à la fois (sou)rire de la manière dont l'oncle Nolte et sa femme éduquent Hélène et y voir une vigoureuse ironie appliquée à la morale petite-bourgeoise protestante (d'autres passages contiennent même une certaine polémique engagée contre la grande bourgeoisie catholique, comme pour contre-balancer un peu les attaques). La morale de l'oncle est un condensé de cette morale petite bourgeoise, au point que la scène située au début du deuxième chapitre («Des Onkels Nachthemd») pourrait servir d'étude à la mission pédagogique du pasteur évangélique 9 . couple âgé de ne plus être ce qu'il a été : certes la jeune fille doit se protéger du mal et, pour cela, prier et encore prier (notons d'ailleurs qu'elle le fait souvent à l'aide d'un chapelet, ce qui n'est pas forcément typique d'une protestante!) et s'inspirer du comportement vertueux des personnes d'un certain âge. Mais si les personnes âgées sont si sages, c'est qu'elles ont leur vie "derrière elles » et ne peuvent probablement rien faire d'autre que prendre une tisane, le soir, sur le confortable canapé de la " gute Stube ", en pantoufles, robe de chambre et bonnet de nuit pour l'homme, et pour la femme en chemise de nuit, quelques papillotes sur la tête. Quelle ironie mais aussi quelle bienveillance dans les traits du dessin ! On remarquera le doigt de l'oncle pointé vers le ciel qui est en quelque sorte mis en parallèle avec le bras d'Hélène maintes fois levé aux cieux! Comme dans la vignette où Hélène découvre une bouteille dans la 
poche de Jean (prononcer : Schang, avec l'accent voulu bien entendu!) La première impression du lecteur est d'ailleurs qu'Hélène est en train de toucher le postérieur de Jean $^{10}$.

12 Mais les conseilleurs n'étant pas toujours les payeurs, on voit bien que l'héroïne ne se comporte pas forcément comme elle aurait envie qu'on le croie. Sa tenue est bien trop coquette et ses allures sont souvent celles d'une fille de la grande bourgeoisie (ou qui voudrait apparaître comme telle). D'une certaine façon, on a affaire ici à la mise en lumière de la difficulté à vivre une morale, voire à la négation de la morale : l'humour aidant, on pourrait peut-être voir ici une allusion à Nietzsche !

Grâce au succès de ses travaux, Busch va publier un recueil de poèmes, Kritik des Herzens, quicerne au plus près les carences morales de ses contemporains; mais il eût mieux fait de rester caché derrière ses figures de papier, car l'indignation petitebourgeoise fut à la mesure de la critique contenue dans les poèmes. Busch sembla avoir compris le message et devint peu à peu l'ermite de Wiedensahl... Dorénavant, il fallait donner au public un héros dont il pût se moquer, mais tranquillement en quelque sorte, sans se sentir directement touché ni concerné, ou tout au moins avec l'alibi de l'image, alibi valable des deux côtés, aussi bien du lecteur que de l'auteur. Arrive donc le personnage de Tobias Knopp : c'est le prototype même du petit-bourgeois de l'époque, appréhendé à son paroxysme... de platitude. Tout ce que Busch discerne en fait de valeurs bourgeoises "saturées", il le met en scène dans cette nouvelle "bande dessinée ", en fustigeant plus ou moins gentiment (et donc: très méchamment!) la bonne conscience du Philistin. Car pour Busch (faut-il voir ici un écho de son éducation protestante encore teintée d'un utilitarisme typique des $\mathrm{XVIII}^{\mathrm{e}}$ et $\mathrm{XIX}^{\mathrm{e}}$ siècles ?), chaque heure perdue est un malheur et Tobias Knopp est bien le héros de ce temps perdu, ses journées ne servant à rien ou à pas grand-chose! Il n'est sur ce point que le représentant d'un monde bourgeois aux valeurs limitées au strict matériel et la première partie de ses aventures (Abenteuer eines Junggesellen) traite de la recherche d'un(e) partenaire, du couple et du mariage bourgeois. Le célibat avait toujours fait peser sur les épaules de Busch un poids social énorme. En privé, cet éternel célibataire pouvait d'ailleurs être plutôt difficile à vivre. Avant sa mort, il a ainsi brûlé toutes les lettres personnelles qui lui avaient été adressées de même que, probablement, ses journaux intimes. On sent chez lui une certaine peur de se livrer et une souffrance qui, souvent, aimerait mieux se cacher. Car à l'époque le célibataire était considéré soit comme un anormal soit comme un dépravé : on connaît bien l'énorme machine mise en place par la société bien pensante du $\mathrm{XIX}^{\mathrm{e}}$ siècle pour combattre l'onanisme et l'homosexualité, dont le célibataire était la plupart du temps soupçonné. D'un point de vue économique et/ou religieux, il paraissait inconcevable de ne pas se reproduire, le mariage devenait ainsi un devoir pour chaque être humain respectable.

Le célibataire Tobias Knopp ${ }^{11}$, qui passe son temps à rendre des visites inopinées à ses amis, ne peut leur faire croire qu'il a la moindre liaison. Lors de ces visites, le lecteur va faire la connaissance de personnages amusants, comme l'ami Mücke, lui aussi célibataire, qui profite de la présence de Knopp pour aller s'amuser avec la serveuse de l'Anguille bleue, un cabaret du coin ${ }^{12}$ ! 

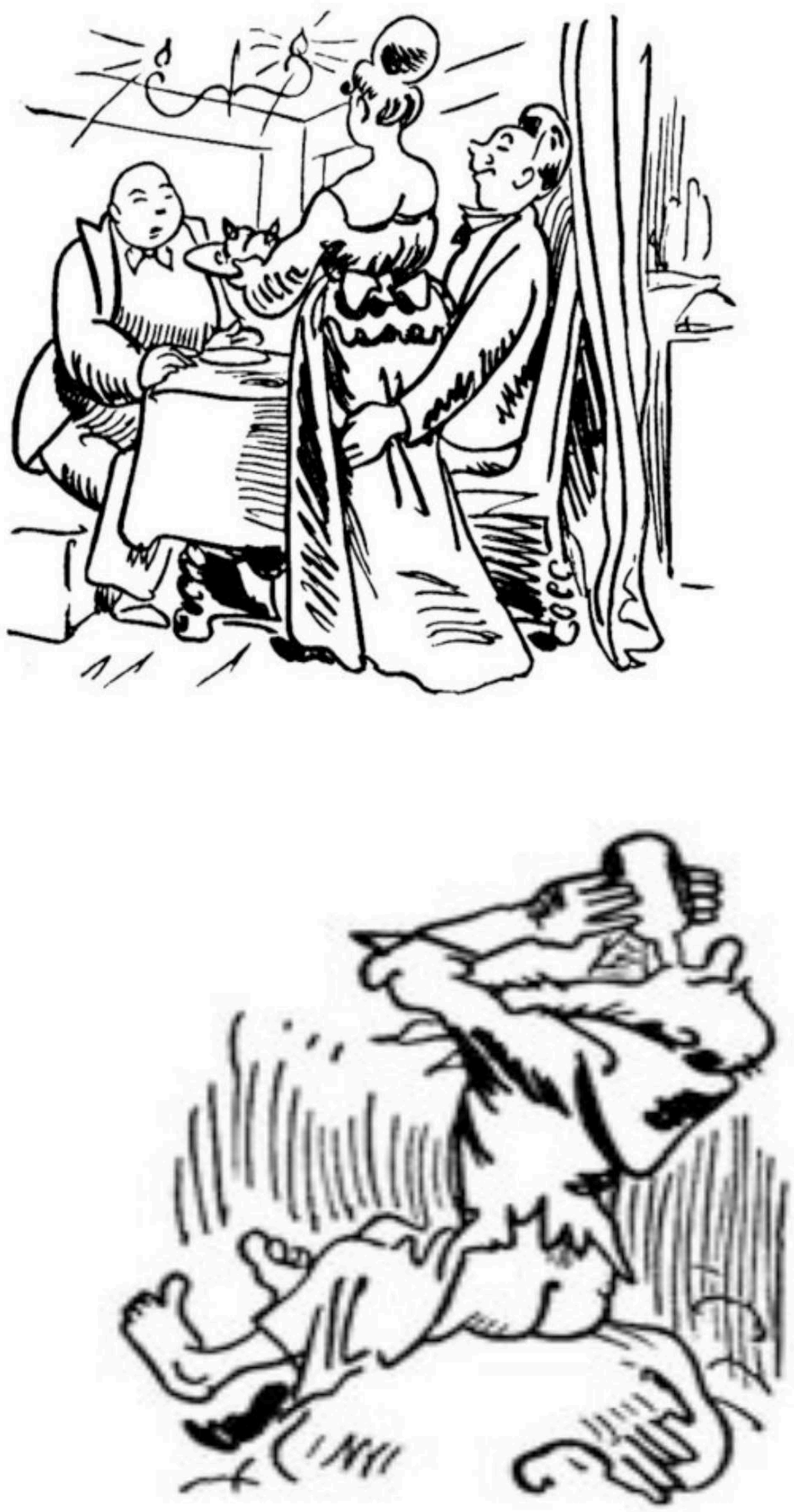

On rencontre également l'ami de jeunesse Knopp Babbelmannan, qui s'est transformé en Philistin en pantoufles, après avoir été autrefois le meneur de jeu de cette bande de joyeux lurons! De rencontre en rencontre, notre ami Knopp continue sa route ( $«$ und begibt (verfügt) sich weiter fort / Bis an einen anderen Ort!»), croisant ainsi l'ami Piepo qui lui présente sa fille Clotilde : cela permet à Busch de jouer avec la polysémie 
du verbe "sich entblättern », car Knopp, maladroit et piètre séducteur, " entblättert sich » comme la rose offerte par la jeune fille s'effeuille (" entblättert sich»). On voit le naï Sauerbrot dont l'épouse vient de mourir et repose dans la pièce d'à côté, ce qui n'empêche pas, au contraire même, les deux hommes de faire la fête - jusqu'à ce que l'épouse apparaisse à la porte, provoquant chez Sauerbrot un arrêt cardiaque! L'ermite Krökel à la repoussante saleté n'incite pas Knopp (et du coup Busch !) à l'imiter, niant les propositions de Schopenhauer en faveur d'une ascèse liée à un utopique éloignement de la société qui permettrait à l'individu d'atteindre une perfection intérieure (ici c'est plutôt à une dégradation extérieure que l'on assiste !)

Donc Busch nous montre plutôt les difficultés que l'homme éprouve à construire du lien et à bâtir de la solidarité. Knopp craint d'être isolé (en tant que célibataire certes mais également en tant qu'humain tout simplement) et ses tentatives pour échapper à ce danger le laissent bien perplexe. On essaie de trouver des solutions, c'est un vaste problème avec lequel chacun(e) d'entre nous s'arrange comme il/elle peut. Après s'être présenté en conquérant du monde, le pauvre Knopp revient entre ses quatre murs où la vue de la bonne Dorothée lui promet un peu de sécurité face aux vicissitudes du destin. Ce port d'attache va être le cadre du tome consacré au couple Knopp.

Certains à l'époque ont vu dans les aventures de ce célibataire, et plus généralement toute l'œuvre de Busch, des traits explicitement pornographiques. Gudrun Schury ${ }^{13}$ souligne l'acharnement de la critique envers la forme de la barbe du pauvre SaintAntoine de Padoue, qui rappellerait certaines parties du corps masculin!

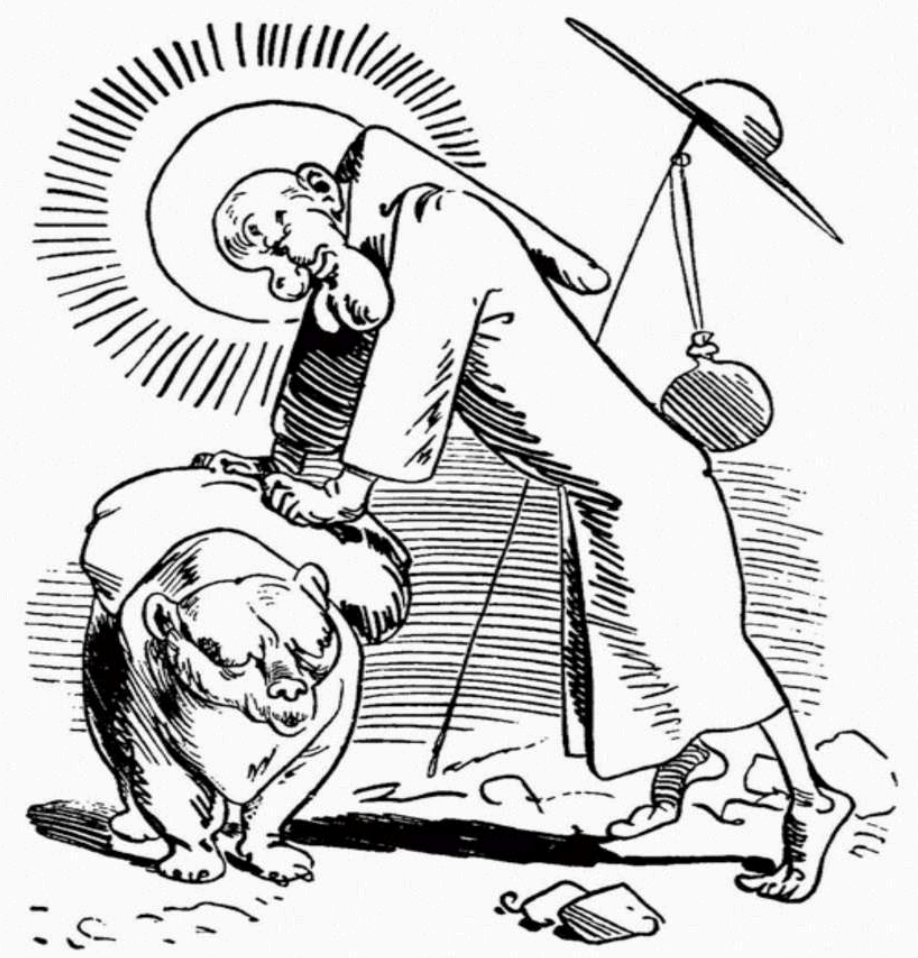

18 Mais s'il y a chez Busch, par moments, des côtés un peu lascifs, s'il lâche parfois des expressions un peu triviales ou simplement suggestives, on cherchera vainement de la pornographie! L'histoire de Tobias Knopp aurait pu s'y prêter mais ce n'est pas le traitement qu'a choisi l'auteur ! Knopp est bien à la recherche d'une femme, à tout prix, car, socialement, "sociétalement ", il ne saurait continuer ainsi. Mais cette obsession 
ne donne lieu à aucune position frivole, à aucune tenue affriolante : Knopp se contente de chercher et, finalement, le message est clair et - trop ? - sage : pourquoi aller chercher loin ce que l'on a sous la main ? Dorothée est là, il l'épouse, et le rideau est tiré sur l'alcôve. Et ce n'est que plus tard que Busch donne une suite à cette histoire, avec l'apparition du bébé Julchen. Ces épisodes vont se vendre très rapidement, ce qui confirme bien une forte attente chez les lecteurs, sensibles au besoin créé par l'auteur. Ce qui montre à quel point les lecteurs de l'époque - et d'aujourd'hui sans doute également - se sentent concernés par le mariage, la vie en couple, la reproduction et l'éducation des enfants.

Les autres histoires de Busch offraient une fin qui frisait parfois le fantastique ou l'inhabituel. Ici rien d'exceptionnel: l'histoire se résume en trois termes, une rencontre, un mariage, une naissance. La trilogie de Knopp est ainsi une sorte d'expérience conduite avec l'aide, voire la complicité du lecteur de l'époque : elle ne traite que du quotidien, surtout dans sa deuxième et troisième partie (Herr und Frau Knopp, Julchen). Monsieur Knopp, bien calé dans son fauteuil à oreilles, fume sa pipe, ou bien tient le bébé sur ses genoux ; on le trouve en train de cirer ses bottes ou promener l'enfant ; Madame Dorothée balaie, sert le café etc.

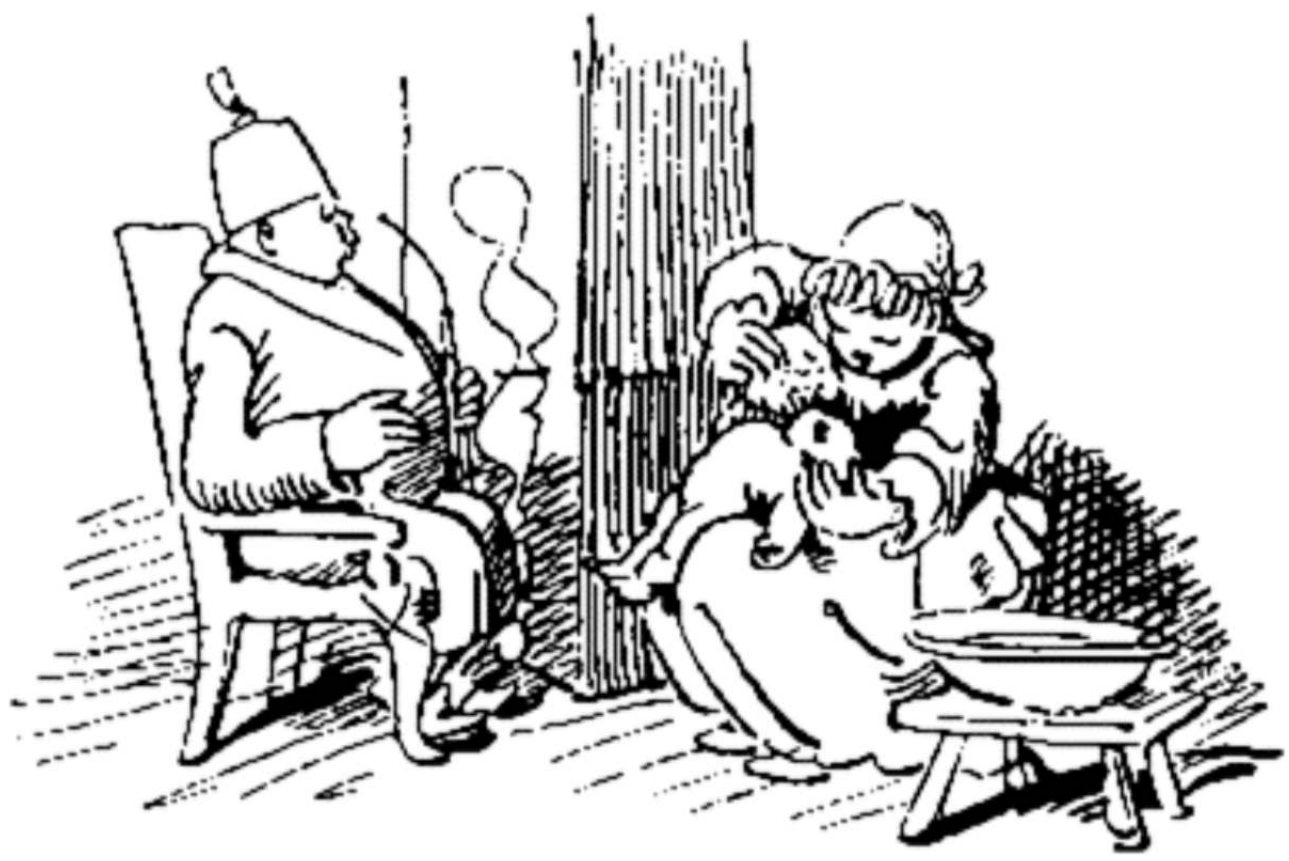

Pas un mot de la vie professionnelle, peu de sorties, pas de voyages. On s'amuse, on mange des crêpes, on fume, on (se) lave, on se rase, on s'habille, on se déshabille, on s'occupe de l'enfant, on surveille les domestiques. Alors certes l'humour est partout. Dans les noms d'abord : le chien de Knopp va se nommer Knipp! Dans les attitudes : car les traits, bien sûr, vont être forcés. Tout ce qui coule, l'eau, la bière, les grogs, le lait, le café, l'encre, la sueur et les larmes, tout coule de façon plus dense que dans la réalité ! Mais rien, à une saine exception près, n'est absurde ou irréel. Remarquons au passage que Busch, célibataire invétéré, est un remarquable observateur de la vie des couples, car il décrit ici en fin de compte une vie dont il n'a aucune expérience. C'est un célibataire connecté à la famille. Et la fin de Knopp - satisfait car il a conduit son enfant au mariage et peut donc mourir en paix - est une fin calme : certes ce n'est pas une fin chrétienne au sens strict du terme puisque Busch fait intervenir une Parque (et non la 
Vierge, ni un ange ni... le diable !), une Parque qui a une verrue sur le nez, image vraisemblablement empruntée à Heinrich Heine - logique et charmante filiation ! -

« In der Wolke sitzt die schwarze Parze mit der Nasenwarze... »

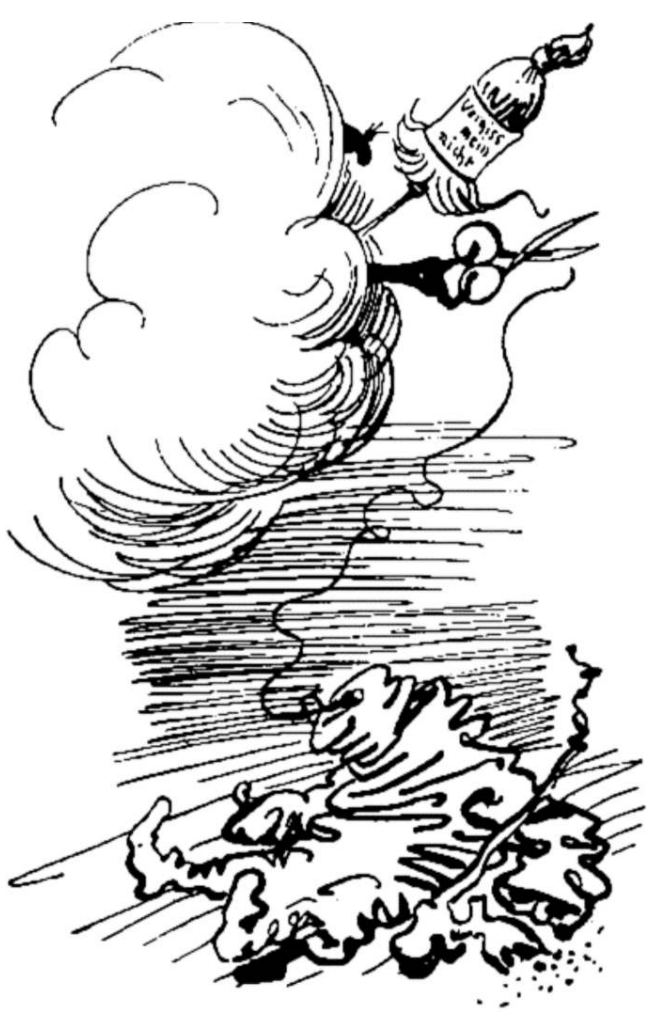

Ce pied-de-nez au monde bourgeois, cet humour constant, cette ironie mordante, tout cela est sans doute teinté d'une certaine amertume. Busch aurait voulu être un esquimau : bienheureux le solitaire, car personne ne peut rien lui faire ! Certes, mais si personne ne peut lui faire de mal, personne non plus ne pourra lui faire du bien ! À l'époque le célibat condamne à la non-paternité (officiellement en tout cas) et cela fait de Busch «l'oncle éternel », une sorte d'oncle errant... L'esquimau sur sa banquise doit donc se consoler comme il peut... De nos jours le célibat est sans doute mieux accepté et ne condamne plus nécessairement à la non-reproduction, mais la question du couple et de l'enfant est omniprésente : sous des formes résolument modernes elle n'en est pas moins douloureuse ou préoccupante pour beaucoup de contemporains. De Max et Moritz en passant par Hélène et d'autres héros, jusqu'à Knopp, les scènes de la vie se déroulent, dégrisant chaque fois davantage le lecteur! Reste ce (sou)rire, jaune parfois. Après un siècle d'utopies non ou mal réalisées, après avoir tenté de vivre des expériences nouvelles, comment ne pas imaginer que la femme et l'homme d'aujourd'hui puissent se reconnaître et se retrouver dans cet instant ironique, cette pause qui permet de se consoler de tous ces - plus ou moins glorieux - petits arrangements avec la vie?! 


\section{BIBLIOGRAPHIE}

\section{Brève biographie}

(http://www.goethe.de/kue/lit/prj/com/pck/ckb/kbb/frindex.html) :

1832 : 15 avril : naissance à Wiedensahl près de Hanovre, aîné de sept enfants

1841 : s'installe chez son oncle, le pasteur Georg Kleine, à Ebergötzen près de Göttingen

1847 : Busch est reçu aux épreuves d'admission de l'école de techniciens supérieurs de Hanovre et étudie la construction mécanique

1851 : études à la Kunstakademie de Düsseldorf (Académie des Beaux-Arts)

1852 : études à la Königliche Akademie der Schönen Künste (Académie royale des Beaux-Arts)

d'Anvers aux Pays-Bas

1853 : est sévèrement touché par le typhus, retour chez ses parents. Busch glane contes et

légendes allemands.

1854 : part à Munich et étudie à l'Akademie der Bildenden Künste (Académie des Beaux-Arts) ;

adhère à l'association d'artistes Jung-München; collaboration à la revue humoristique Fliegende Blätter

1865 : publication de l'histoire illustrée Max und Moritz (« Max et Moritz »)

1868 : part à Francfort où vit son frère Otto ; rencontre le philosophe Arthur Schopenhauer et Johanna Kessler

1879 : retour dans son village natal de Wiedensahl ; publication de quelques œuvres telles que Fipps der Affe (Fipps le singe)

1898 : part à Mechtshausen dans le Harz (près de Seesen) ; publication du recueil de poèmes $\mathrm{Zu}$ guter Letzt (À la fin des fins)

1908 : meurt le 9 janvier d'un arrêt cardiaque à Mechtshausen

\section{Sources}

Wilhelm Busch, Sämtliche Werke

Sämtliche Werke und eine Auswahl der Skizzen und Gemälde in zwei Bänden Bertelsmann Verlag München, 1982.

\section{Études critiques}

Karl Anlauf, Der Philosoph von Wiedensahl, Der völkische Seher Wilhelm Busch, Buchmeister Verlag, Berlin, 1939

Hans Balzer, Nur was wir glauben, wissen wir gewiß, Der Lebensweg des lachenden Weisen Wilhelm Busch, Evangelische Verlagsanstalt, Berlin, 1957 
Ulrich Beer, Wilhelm Busch, Lausbub - Lästermaul - Lebensweiser, Verlagsbuchhandlung, G. Schumacher, Rehburg-Loccum, 2000

Friedrich Bohne, Wilhelm Busch, Leben - Werk - Schicksal, Fretz \& Wasmuth Verlag, Zürich, 1958

Michaela Diers, Wilhelm Busch, Leben und Werk, Deutscher Taschenbuch Verlag, München, 2008

Gerhard Flügge, Wilhelm Busch, VEB Bibliographisches Institut, Leipzig, 1967

Herbert Günther, Der Versteckspieler, Die Lebensgeschichte des Wilhelm Busch, Union Verlag, Stuttgart, 1991

Joseph Kraus, Wilhelm Busch, Rowohlt Taschenbuch Verlag, Hamburg, 2007

Dieter P. Lotze, Wilhelm Busch, Leben und Werk, Belser Verlag, Stuttgart, 1982

Otto und Hermann Nöldeke, Wilhelm Busch, Buch Verlagsanstalt Hermann Klemm, BerlinGrunewald, 1930

Gert Ueding, Wilhelm Busch, Das 19. Jahrhundert en miniature, Insel Verlag, Frankfurt am Main, 2007

Gudrun Schury, Ich wollt, ich wär ein Eskimo, Das Leben des Wilhelm Busch, Aufbau Verlag, Berlin, 2007

Hans Dieter Schwarze, Mein lieber Wilhelm! Unverhoffte Begegnungen mit Wilhelm Busch, Schneekluth, München, 1982

Eva Weissweiler, Wilhelm Busch, Der lachende Pessimist, Kiepenheuer \& Witsch, Köln, 2007.

\section{NOTES}

1. http://www.bamako.diplo.de/Vertretung/bamako/fr/06/Deutsche__Literatur/ Wilhelm__Busch__seite.html, site consulté pour la dernière fois le 20/08/2010.

2. Cf. brève biographie en fin d'article; on pourra se reporter également à l'ouvrage: Kraus, Joseph, Wilhelm Busch, rororo, Hambourg, 2007.

3. http://de.academic.ru/pictures/dewiki/77/MaxMoritz.jpg, site consulté pour la dernière fois le $20 / 08 / 2010$.

4. http://www.bamako.diplo.de/Vertretung/bamako/fr/06/Deutsche__Literatur/

Wilhelm__Busch__seite.html, site consulté pour la dernière fois le 20/08/2010.

5. Schury, Gudrun: Ich wollt, ich wär ein Eskimo. Das Leben des Wilhelm Busch, éditions Aufbau, Berlin, 2007.

6. Cf. entre autres: Diers, Michaela, Wilhelm Busch, Leben und Werk, Deutscher Taschenbuch Verlag, Munich, 2007 ; Schury, Gudrun : "Ich wollt, ich wär ein Eskimo... ", op. cit. ; Weissweiler, Eva : Wilhelm Busch. Der lachende Pessimist, éditions Kiepenheuer \& Witsch, Cologne, 2007.

7. http://www.jadu.de/jadukids/maerchen/max_und_moritz/diashow/Bilder/seite20_1_jpg.jpg. 8. À ce propos, on pourra consulter l'article http://www.universalis.fr/encyclopedie/wilhelmbusch/site vérifié et consulté pour la dernière fois le 20/08/2010.

9. http///www.wilhelm-busch-seiten.de/werke/Hélène/kapitel02.html: « Hélène! » - sprach der Onkel Nolte -/ Was ich schon immer sagen wollte!/ Ich warne dich als Mensch und Christ:/ Oh, hüte dich vor allem Bösen!/ Es macht Pläsier, wenn man es ist,/ Es macht Verdruß, wenn man's gewesen! »/ «Ja leider! » - sprach die milde Tante -/ « So ging es vielen, die ich kannte!/ Drum soll ein Kind die weisen Lehren/ Der alten Leute hochverehren!/ Die haben alles hinter sich/ Und 
sind, gottlob! recht tugendlich!/ Nun gute Nacht! Es ist schon späte!/ Und gutes Lenchen, bete, bete! »

10. http://wilhelm-busch-steinen.de/werke/Hélène/kapitel110.html.

11. Cf. http://gutenberg.spiegel.de/?id=5\&xid=3014\&kapitel=1\#gb_found, site consulté pour la dernière fois le 20/08/2010.

12. http://www.zeno.org/Literatur/M/Busch,+Wilhelm/Bildergeschichten/ Abenteuer+eines+Junggesellen/Freund+M\%C3\%BCcke.

13. Gudrun Schury, op. cit., p. 171-172.

\section{RÉSUMÉS}

Wilhelm Busch (1832-1908) est incontestablement un des fondateurs de la BD moderne. Il est très populaire dans les pays de langue allemande, un peu moins peut-être en France, mais des œuvres comme Max und Moritz, Die fromme Helene ou Tobias Knopp ont contribué à lui assurer dans le monde un succès marqué. Les thèmes abordés dans ces petites histoires composées de textes rimés et d'illustrations s'inspirent de la vie quotidienne à la campagne ou dans de petites villes. On voit des garnements jouant des tours aux artisans ou aux paysans, des jeunes filles élevées dans la religion qui, parce qu'elles ont cédé à la tentation, seront, comme les joyeux farceurs, sévèrement punies. La morale est sauve. Succès garanti également pour les aventures d'un vieux garçon, Tobias Knopp, que l'on suit dans ses tribulations de célibataire, jusqu'au mariage, à la naissance de son enfant et à sa mort. Rien que de très quotidien, avec quelques rares incursions dans l'extraordinaire ou le fantastique. La critique envers la société bourgeoise (petitebourgeoise) du XIX siècle, est forte, acérée, mais le désarroi de l'auteur, lui-même vieux garçon invétéré, se ressent. En quoi ces histoires peuvent-elles encore nous toucher aujourd'hui ? Audelà des informations qu'elles livrent sur une société ancrée dans un espace-temps bien précis, l'Allemagne de la deuxième moitié du XIX $\mathrm{x}^{\mathrm{e}}$ siècle, elles nourrissent un questionnement sur la vie qui est encore (en grande partie et sous d'autres formes parfois) d'actualité. Et la qualité du dessin, le soin apporté aux détails, la vigueur du trait, la dynamique des personnages, tout cela ne peut que rester gravé dans l'esprit du lecteur.

Wilhelm Busch (1832-1908) ist zweifellos einer der Begründer des modernen Comics. Er ist in den deutschsprachigen Ländern sehr beliebt, in Frankreich kennt man ihn wohl weniger, dennoch haben Werke wie Max und Moritz, Die fromme Helene oder Tobias Knopp zu seinem internationalen Erfolg beigetragen. Seine kleinen, aus Reimen und Illustrationen bestehenden Geschichten finden ihre Anregungen im täglichen Leben auf dem Lande oder in Kleinstädten. Seien es nun freche Schlingel, die Handwerkern oder Bauern Streiche spielen oder junge fromme Mädchen, die ernsthaft bestraft werden, weil sie der Versuchung nachgegeben haben - die Moral kommt bestimmt. Ebenfalls ein Erfolgsgarant ist der alte Jüngling, Tobias Knopp, dessen Leben der Leser angefangen vom Leid eines Unverheirateten über seine Heirat, die Geburt seines Kindes und schließlich bis zu seinem Tode begleitet. Lediglich das ganz und gar Alltägliche mit nur spärlichen Ausflügen ins Ungewöhnliche oder gar Fantastische. Die Kritik an der bürgerlichen Gesellschaft (Kleinbürgertum) des 19. Jahrhunderts ist scharf und nachdrücklich und doch spürt man die Hin-und Hergerissenheit des Autors, der selbst ein eingefleischter Junggeselle ist. Inwieweit können uns diese Geschichten heute noch ansprechen? Sie liefern nicht nur Informationen über eine fest zeitlich und räumlich verortete Gesellschaft, nämlich die im 
Deutschland in der zweiten Hälfte des 19. Jahrhunderts, sondern sie animieren auch Fragestellungen über das Leben, welche zum großen Teil so oder so ähnlich immer noch aktuell sind. Letztendlich ist auch die Qualität der Zeichnungen zu erwähnen, die mit ihrer Liebe zum Detail, ihren kraftvollen Strichen und der Dynamik der Charaktere unweigerlich Spuren in den Köpfen der Leser hinterlassen müssen.

Wilhelm Busch (1832-1908) is unquestionably one of the founders of the modern comic strip. He is very popular in German-speaking countries, and a little less so in France perhaps, but works such as Max und Moritz, Die fromme Helene or Tobias Knopp have assured him considerable success throughout the world. The subjects dealt with in these tales composed of rhyming text and illustrations take their inspiration from everyday life in the countryside or small towns. We see rascals playing tricks on craftsmen and peasants, or girls with a religious upbringing who, because they have yielded to temptation, end up being severely punished, just like the merry pranksters. Moral standards remain intact. Success is also ensured for the adventurers of a bachelor, Tobias Knopp, whose trials and tribulations we follow from bachelorhood through to his marriage, the birth of his child and his death. It's all everyday stuff, with some rare forays into the extraordinary or fantastic. The criticism of bourgeois society (the lower middle classes) of the 19th century is fierce and biting but the confusion experienced by the author, himself a confirmed bachelor, can be felt. In what way do these tales still concern us today? Beyond the information that they provide about a society anchored in a very specific setting in terms of space and time, the Germany of the second half of the 19th century, they provide material for reflection on life which is (to a large extent and sometimes in other ways) still of relevance. The quality of the drawings, the care taken on small details, the firmly drawn lines and dynamism of the characters cannot but remain engraved in the mind of the reader.

\section{AUTEUR}

\section{RICHARD PARISOT}

Université de Franche-Comté (Besançon) 